\title{
An Initial Model for Zero Defect Manufacturing
}

\author{
John Lindström ${ }^{1, *, \dagger}$, Petter Kyösti ${ }^{2,+} \mathbb{C}$, Wolfgang Birk ${ }^{2,3,+} \mathbb{C}$ and Erik Lejon ${ }^{4,+}$ \\ 1 Combitech AB, Varvsgatan 31 7tr, 97236 Luleå, Sweden \\ 2 Luleå University of Technology, 97187 Luleå, Sweden; petter.kyosti@ltu.se (P.K.); \\ wolfgang.birk@ltu.se or wolfgang.birk@predge.se (W.B.) \\ 3 Predge AB, Varvsgatan 11, 97236 Luleå, Sweden \\ 4 Gestamp HardTech AB, 97245 Luleå, Sweden; elejon@se.hardtech.com \\ * Correspondence: john.lindstrom@combitech.se; Tel.: +46-73-4375776 \\ + These authors contributed equally to this work.
}

Received: 9 June 2020; Accepted: 25 June 2020; Published: 1 July 2020

\begin{abstract}
This paper investigates an initial model for Zero Defect Manufacturing (ZDM) using a cost function where the operation and condition of a production process are reflected, and the quality of the output/product and the production process (as well as safety aspects) can be considered. The outset of the study is based on empirical data collected from five manufacturing companies, and proposes an initial model for ZDM with an Industry 4.0 perspective. The initial ZDM model has a generic setup for a real-life system and its replication as a digital twin using system models based on a representation of a generic production process with its connected control system, and potential interconnections between unit processes. It is based on concepts from system theory of dynamic systems and principles from condition monitoring and fault detection. In that way the model is deemed as highly generalizable for manufacturing and process industry companies as well as for some critical infrastructures with production and distribution systems. The proposed model with its cost function setup is analyzed and discussed in the context of ZDM. It is concluded that production processes in the manufacturing and process industry can be made more intelligent and interoperable using this approach. Improved sustainability, competitiveness, efficiency and profitability of companies are foreseen welcomed secondary effects. Finally, the proposed ZDM model further develops the ZDM by adding to it a systematic approach based on a solid mathematical foundation.
\end{abstract}

Keywords: digital twin; Industry 4.0; intelligent; model; sustainable; system model; zero defect manufacturing (ZDM)

\section{Introduction}

Zero Defect Manufacturing (ZDM) was coined during the later part of the 1980s, with the intent to minimize (or cancel) the defects in the output from various production processes. It is sprung out of the early work by Stewart and Deming (cf. [1]) and later became an important component of Total Quality Management (TQM). The main challenges associated with ZDM have been to integrate large amounts of information from many sources, the need to develop advanced technology and methods, the modeling of ZDM, and timely (on-line or in real-time) computing [2]. These issues have also been confirmed by a recent $Z D M$ research study with six participating manufacturing and process industry companies [3]. There, the needs and desired effects from applying ZDM are also elaborated on.

The aim of the paper is therefore to propose, analyze and discuss an initial model for ZDM, with a clear Industry 4.0 perspective, applied to the production in the manufacturing and process industries. It is based on the third cycle (out of five planned) in an action research effort conducted at five companies, mainly in manufacturing with both discrete- and continuous sub-processes as part of the main production process. 
Our overarching view on ZDM is that errors and flaws will always exist and impact the production and its output, but that these can be minimized and detected quickly on-line, and that no production output deviating from specification should be able to reach the next step in a value-chain or eventually the end-customer. This requires a different mindset compared to separated or local monitoring of process parameters, production equipment and batch quality control, like, for instance, sampling three out of a thousand outputs. A well established approach in the field of automatic control is to use dynamic system models to establish estimation schemes for faults, qualities and process conditions [4], which could also be suited for ZDM and is an integral part of the proposed method. Thus, the previous barriers for adoption of ZDM and the pre-requisite innovations are now starting to be overcome at a fast pace.

The foreseen benefits of a successful introduction of ZDM include: reduced costs, increased sustainability (by reduced energy and resource consumption), increased Overall Equipment Efficiency (OEE) and more predictable product quality, which result in increased customer confidence. In addition, more information will be available about the produced products, increasing the value of the product by enabling customers to include it in their own ZDM system and to achieve similar effects. As a result, the ZDM system can serve as a data infrastructure and a technological enabler for eco-innovation $([5,6])$, Six Sigma and Lean.

ZDM, as investigated in previous research $[7,8]$, combines and integrates the following seven areas:

- Monitoring of process parameters;

- Collaborative manufacturing - which includes sharing of manufacturing data without media breaks along the supply chain;

- Continuous quality control - which includes measurement of input to the process and measurement of output from the process with automatic sorting;

- On-line predictive maintenance;

- Data storage, analytics and visualization;

- Re-configuration and re-organization of production;

- Re-scheduling of production.

As stated in [8], the reason for combining and integrating the seven areas is that they together can achieve a better result. However, sometimes the issues may not be found, since all parameters and activities are not measured or monitored.

Concerning related work, Konrad et al. [9] outline an intelligent manufacturing process control, based on a thorough understanding of the production process, comprising inline quality control, quality thresholds, and process parameter effects on output, which can be applied in the effort to achieve ZDM. Further, Lee et al. [10] propose a Cyber-Physical Systems (CPS) architecture for Industry 4.0-based manufacturing systems composed of the five Cs: connection, conversion, cyber, cognition and configure, with associated applications, methods and techniques, in order to achieve better product quality and more intelligent and resilient manufacturing equipment. In addition, Lin et al. [11] suggest the development of smart manufacturing in an Industry 4.0 context using a five-stage strategy to enhance the yield and assurance in manufacturing platforms. All of this related work points towards the same direction, but using somewhat different means and tools to move towards more competitive, intelligent and sustainable manufacturing.

Today, the ZDM-drivers are, for most manufacturing and process industry companies, to become more competitive, profitable, sustainable and intelligent with related effects, such as: lower costs, lower energy consumption (per output), lower level of wasted materials and scrapped output, faster production processes, better overview of the production process, improved planning ability, production systems robustness and resilience, higher level of availability, and improved level of output quality $[2,3,8]$.

Thus, the problem addressed is how to start combining and integrating data and knowledge into an initial model for ZDM, which can be adapted and detailed for most scenarios in the manufacturing and process industries. Subsequently, one part of the purpose is to provide an initial model for those 
companies who want to move towards more sustainable and intelligent production using a ZDM approach. The second part of the purpose is to develop ZDM as a concept with a systematic approach using a fundamental mathematical description.

Figure 1 visualizes how a ZDM approach can be implemented. Further, Figure 1 outlines that the ZDM system model and the control system constitute the central hub for information, connecting pre- and post-measurements of the production process step with on-line process and maintenance parameters as well as historical measurements into a central model. The model's output depends on user knowledge and input, and provides decision support as output.

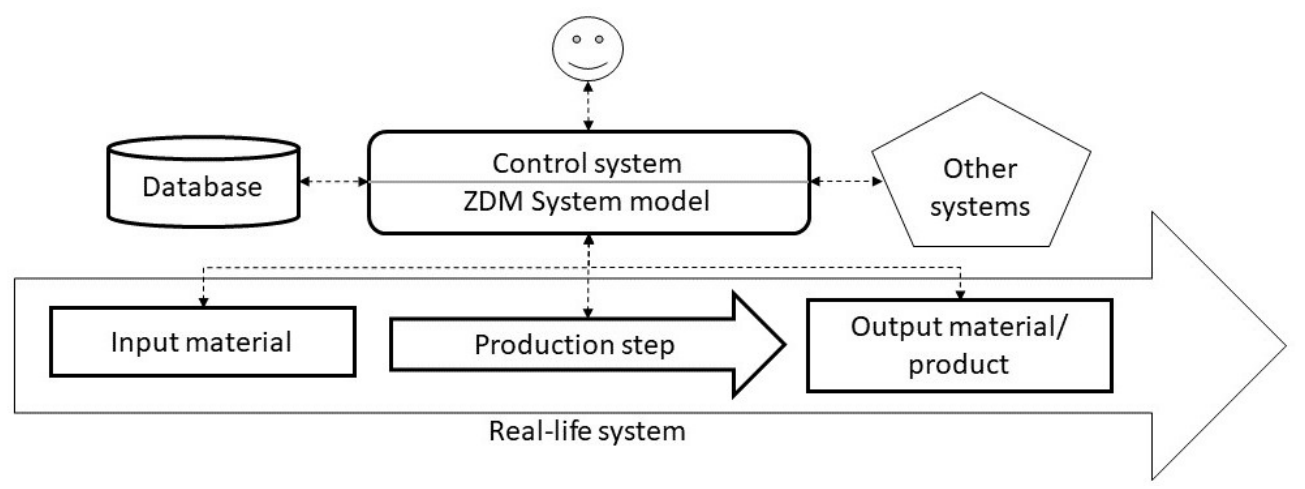

Figure 1. Diagram showing information flow in a generic production process step with an integrated control system.

Following the introduction, there are sections on related work, research methodology, findings, analysis and finally discussion and conclusions.

\section{Related Work for Zero Defect Manufacturing}

The ZDM-related work and state-of-art are outlined below in terms of background, usage and interest. As ZDM is a multi-disciplinary concept, the literature review covers a number of related research fields and brings them together.

\subsection{Background of ZDM}

One of the forefathers of the modern quality management was W. Edwards Deming, who, together with Walter Stewart, started to contribute already in the 1920s to what later was to become ZDM. Deming worked a lot with the Japanese after WW2, and the Japanese picked up a lot from this concerning quality management and continuous never-ending improvement [1] as a management philosophy. Deming went back to the USA in the 1980s and lectured on quality management, and is credited as one of many who have contributed to the concepts of just-in-time, the Toyota Production Model, and later also Lean and Six Sigma.

TQM was developed during the 1980s and was a departure from traditional management thinking, as all departments were given objectives and should improve their business and activities. TQM started early to address sustainable development [12], which later became an important corner-stone. However, TQM never became a success, perhaps due to the top-management control and lack of connection with quality and cost, which limited its effect. TQM also had as its objectives to achieve "zero defects" and "do it right the first time", which probably steered the focus to these too much (and they were hard to achieve then). Thus, this may have hindered new and radical changes in the development of new products and services [13].

ISO 9000, and later also Six Sigma, started to break through during the 1990s. ISO 9000 is a general framework for quality and Six Sigma concerns variations in products. Some corporations have made substantial savings as a results of using Six Sigma [14]. However, critics posit that Six Sigma is not really anything new and builds on classic quality management, sets too-low requirements for product 
quality, requires consultants who do not have knowledge about the business, relies on sometimes weak statistical correlations, and is not able to handle new products and disruptive technologies.

ZDM was coined in 1987 as part of TQM, and is thus a well-researched area that has started to gain more interest lately, as it now is more feasible to set "zero defects" as an objective. This development is due to the recent technological progress and implementation of Industry 4.0 [10] with Big Data and data mining, real-time analysis of streaming data, digital twins, advanced simulation, automated planning, cloud services, CPS, smart sensor networks and the Internet-of-Things (IoT). During recent years, Artificial Intelligence (AI) and Machine Learning (ML) have together with the aforementioned made it possible to autonomously monitor large amounts of diverse data in order to predict, detect and trace the origin of errors. Further, the need to understand business processes and the production systems and equipment part of these has increased due to the need to be able to model them. The modeling is required for data analysis, simulations and predictions [15,16]. Without the models, the outcome of the analytics will be meagre. In addition, the quality of the data used needs to be ensured in order to get output, i.e., correlations and decision support, with a high level of confidence to really be able to trust the outcome. An information architecture is commonly required to get a grip on, and overview of, the data to use.

ZDM can, according to [2], decrease the cost of a company's resources allocated to management and post-processing of defective products, as the ZDM-thinking builds on the fact that no defect element should be present in a production process at any time. "Defect elements" concerns all that does not bring any value to the product, e.g., defective machines and tools, bad materials or inputs or inefficient workers. Dong et al. [17] posit that the production that is scrapped (i.e., costs only) can be decreased through ZDM. Further, elements outside of the own production process should continuously be scrutinized and improved. The approach can be motivated through improved safety and customer satisfaction, which increase customer loyalty and improve a company's financial outcome [18]. Previously, the ZDM concept has been implemented mainly on parts of production processes due to technical limitations. However, the development of the Industry 4.0 concept enables:

- The ZDM concept to be more easily implemented, due to the availability of the necessary amounts of data (Big Data) required for techniques such as ML to work (cf. [19-22]). However, a much better integration and coordination of the capacity of each technique or method used is still required, and in addition

- The equipment used to monitor and save data used to be expensive-which contributed to the fact that many corporations did not embrace ZDM (cf. [23]). This situation has changed since a few years back with increasingly cost-efficient computing power and data storage, which together with the new emerging technology, methods and techniques makes the ZDM concept possible to approach and implement (in both both economical and technical aspects).

Konrad et al. [9] highlight the need to have inline quality monitoring and control of process parameters in order to achieve intelligent manufacturing process control, whereby out-of-specification items can be detected and removed from the production process. They further state that it is necessary to understand the process, quality thresholds, and which process parameters have certain effects on the final result of the output. This work is in line with the most recent contributions to ZDM from our research group, where we intend to integrate and combine a number of areas and disciplines from input to output in the production process, in order to reach zero defects; these are, e.g., [7,8,24-26]. Our view on ZDM is that we do not consider it is possible to reach zero defects in the production process, but that the most important objective is that no outputs or products outside of specifications can reach the next step in a value chain or the customer.

\subsection{Usage of $Z D M$}

Since ZDM was coined in 1987, data regarding the areas where ZDM has been investigated have been presented. According to Psarommatis et al. [2], about $80 \%$ of the ZDM investigations 
concern detection (of errors or faults). This is only to be expected, as detection is required in order to take on more ambitious actions, such as the additionally complex repair, prediction and prevention, which require integration with other information systems. Further, it is obvious that some industries have embraced ZDM more than others, such as the semi-conductor industry [11]. The semi-conductor industry is relatively young and can quite easily decide if an output is OK or not, whereas other parts of manufacturing and process industries need to use multi-measurement techniques and methods and 3D measurements in order to ensure zero defects [9]. Further, the most common application of ZDM seems to be in the middle or at the end of production processes, which suggests that ZDM is often used as an additional quality management system for outputs/products. According to [2], the most common process steps where ZDM is applied are: manufacturing of wafers (12.6\%); additive manufacturing $(8.3 \%)$; moulding/casting $(8.0 \%)$; welding $(5.3 \%)$; assembly $(4.3 \%)$; and production of TFT /LCD panels (3.3\%). In the above, the most common errors or faults searched for are: cracks, pores, imperfections and internal stress in materials.

\subsection{Interest in $Z D M$ Is Increasing}

Interest in ZDM as a concept is increasing, which is possible to measure from the number of scientific articles that concern monitoring, measurement techniques and methods, Big Data and data analysis. In particular, countries with high labor costs (such as in Northern Europe and the USA) show a growing interest in ZDM and delivery of products within specifications. A number of communities and groups have emerged during recent years, such as the European Union (EU)/Horizon 2020-funded Zero Defects Manufacturing Platform (ZDMP) (https://www.zdmp.eu/), in order to create co-operation spaces, knowledge exchange, and project building and brokerages with stakeholders within ZDM. The EU and its Horizon 2020 "Factories of the Future" has had a number of calls for projects concerning ZDM or closely related areas or concepts.

\subsection{Relation to Condition Monitoring and Fault Detection}

The quality of products depends on the condition of the production system and the used equipment. In the field of automatic control as well as operation and maintenance, establishing information on the condition of a system in terms of, e.g., wear, tear and faults, has been studied intensely for a long time [4].

Taking a system theoretic approach to fault detection has also been done successfully and rendered in real-life implementation, see for example the model-based control and gas leakage detection system discussed in $[27,28]$, which is online since 1999. A further development is also the combined approach of condition monitoring considering operational characteristics, as proposed in [29].

It is the belief of the authors that the combination of a system theoretic approach with the ZDM concept has not been reported about and has potentially not been investigated before.

\section{Research Approach}

The research in this study has been based on an in-depth qualitative study using action research spanning five manufacturing companies, which all have both discrete and continuous parts in their production processes, located in Sweden. It would have been possible to use other iterative, agile or spiral models having iterated refinement of the research results. However, action research was deemed as a good fit due to its flexibility and the possibility to use various data collection and analytic methods.

The companies involved in the study were from the vehicle parts, vehicle, pre-fabricated building and laminate floor industries. The names of the companies, except Gestamp HardTech AB, which is a producer of press-hardened vehicle parts, are kept confidential as agreed. All of the companies have R\&D capabilities within Sweden, which was a requirement to participate in the study.

The research targeted in this paper is the third cycle's first and second phases, i.e., diagnosing and planning action, of an action research [30] effort where the researchers have had the roles of external experts and consultants and internal experts. The total research effort, in order to reach "full" ZDM, 
is foreseen to be 5 cycles. This research was conducted as part of the first (out of three) steps in the Swedish Innovation Agency VINNOVA's program for challenge-driven innovation. Further, each cycle includes 4 phases as outlined below.

Prior to this third cycle, two cycles of action research have been conducted at Gestamp HardTech $\mathrm{AB}(\mathrm{cf} .[7,8])$. A literature review, concerning finding new ideas, approaches and research since the second cycle ended, was part of the first phase, and its result was used as input to the diagnosis. Action research has been defined as "a participatory, democratic process concerned with developing practical knowing in the pursuit of worthwhile human purposes, grounded in a participatory worldview which we believe is emerging at this historical moment. It seeks to bring together action and reflection, theory and practice, in participation with others, in the pursuit of practical solutions to issues of pressing concern to people, and more generally the flourishing of individual persons and their communities" [30]. The characteristics of action research are:

1. that action researchers act in the studied situations, and

2. that action research involves two goals. The goals pertain to solving the problem (the role of the consultant) and making a contribution to knowledge (the role of the researcher); further, that action research requires interaction and cooperation between researchers and the client personnel and, finally, that action research can include all types of data gathering methods [31].

In accordance with [32], the action research approach encompasses 4 phases: diagnosing, planning action, taking action and evaluating the action in relation to a certain context and specific purpose.

The third cycle's first and second phases were completed with an iterative and reflective case management methodology. The data pertaining to the first phase, i.e., mainly technical, methodological and organizational needs for change, were collected during a workshop or site visit [33] at each of the companies (commonly involving 3-8 key respondents from the R\&D, production and maintenance departments at each workshop) and a following semi-structured open-ended interview ([34,35]) with key respondents (e.g., R\&D managers, development managers, IT and automation engineers, and senior researchers or development engineers) at each company. The workshops, site visits and interviews were conducted during 2019. The respondents were well aware of and knowledgeable regarding production systems and equipment, predictive maintenance, quality control, sensors and input-output measurements.

Semi-structured interviews with open-ended questions $([34,35])$ were used after the workshops and site visits to collect data. This allowed the respondents to give detailed answers and the possibility to add extra information where deemed necessary [36]. The duration of the interviews was between one and three hours. In order to reduce response bias, the respondents came from various parts of the organization as well as different levels, i.e., strategic, tactical and operational units. In order to strengthen the validity of the study, data were continuously displayed using a projector during the interviews, allowing the respondents to immediately read and accept the collected data. After that, the collected data were analyzed using matrices (cf. [37]) and the outcome of the planning action efforts was summarized into causal relational graphs (cf. [37]). The analyzed data were finally summarized into a number of figures comprising the diagnosis in terms of integration and combination needs embodied in an initial model for ZDM as well as a high-level plan for action in order to start to adapt and detail the initial model for each company. The design criteria for the action research were: simplicity, low cost, robustness, high-quality output and future-proofing. The design criteria will not be formally evaluated (as the action in the third cycle has not yet been conducted) in this paper. However, the design criteria will be discussed in the last section of the paper to learn whether the outcome points "towards profitable, sustainable, and intelligent production systems" and "ZDM".

\section{Findings-An Initial Model for Zero Defect Manufacturing}

The outset for describing a model for ZDM is taken from the understanding of how a system's behavior can be explained. Conceptually speaking, an envelope for the system behavior can be 
defined as depicted in Figure 2. Obviously, domain knowledge will aid in explaining the system behavior to a certain degree. The domain knowledge can comprise first principle models of the system, experiences of practitioners and engineers, or observations, just to mention a few. Complementary to the domain knowledge, some data are acquired by sensing at and surrounding the system, together with records from observations, as well as operational, maintenance and design requirements imposed on the system.

From Figure 2 it is clear that data alone will only be able to explain the parts of the system behavior that have been encountered in reality and, thus, are examples of the system behavior.

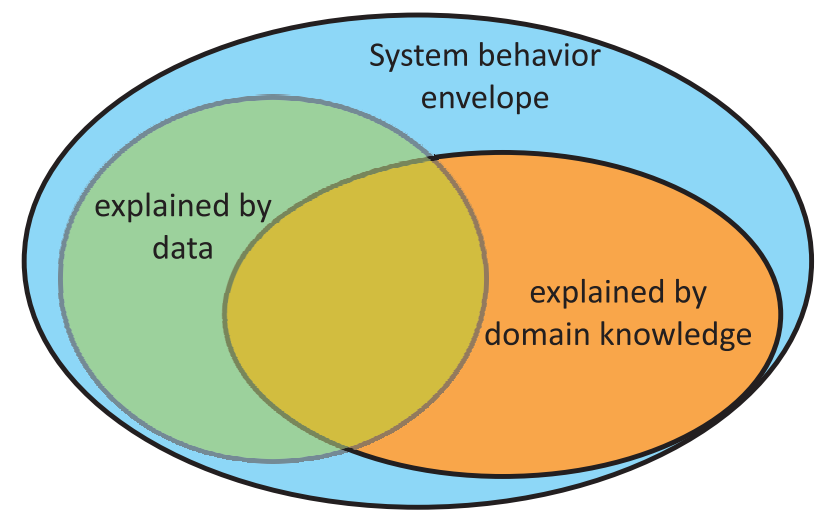

Figure 2. Understanding a system's behavior on the basis of domain knowledge and data.

\subsection{Generic Framework for a Model of ZDM}

Combining operational and maintenance aspect into one framework has been promoted by [29]. It actually means that the system is studied from the perspective that the operation affects degradation and vice versa. The idea is to use this system theoretic approach also in ZDM.

In order to systematically study the system behavior, the real-life context of a system as depicted in Figure 3 needs to be considered. There, the real-life system is affected by Manipulated Variables (MV) and Disturbance Variables (DV). The latter are exogenous effects that might be known through measurements, sometimes only on a qualitative level, or not known at all. The system then generates measurable outputs that need to adhere to certain specifications denoted Controlled Variables (CV) and other measurable outputs denoted Process Variables (PV). Moreover, internal system states, not necessarily measurable, are also categorized as PVs.

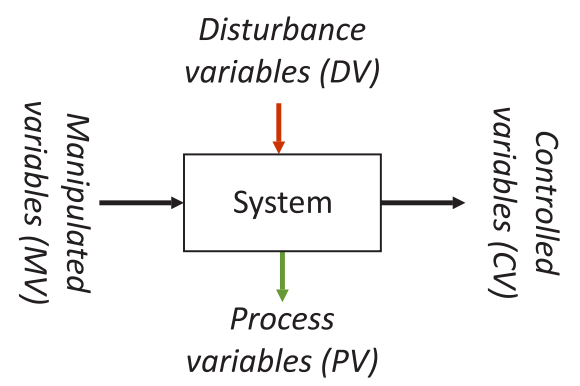

Figure 3. Principal sketch for a system in a real-life context.

Combining domain knowledge with data-driven modeling enables a more complete understanding of the system behavior and therefore aids in extending domain knowledge. Similarly, domain knowledge reflected by first-principle models can enrich data-driven modeling through the generation of synthetic data. In this way the usual use for simulation, monitoring and subsequent analytics is complemented. 
To conclude, the most effective way of determining a model for the system is achieved by combining data-driven and first-principle models in conjunction. Henceforth, the term system model will be used.

While system models enable the systematic design, operation, optimization, monitoring and control of a system, the validity is a critical aspect when relying on the models. In Figure 4, the system model is shown in a real-life context, where it is used in parallel with the real-life system. All variables denoted by $\hat{\boldsymbol{}}$ represent the estimate of the actual value of a variable. The estimate might be the result of a filtering, smoothing or calculation, and might include qualitative judgements. For the MVs $u$ it is assumed that those are known and thus no estimate is needed. Thus, DVs are denoted as $d$ and $\hat{d}$, PVs are denoted as $x$ and $\hat{x}$, and CVs are denoted as $y$ and $\hat{y}$.

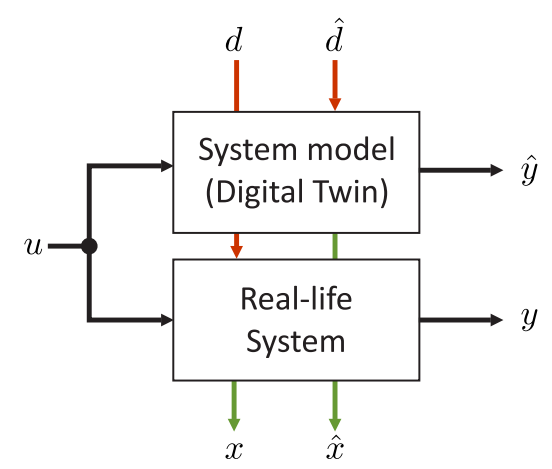

Figure 4. Generic setup for a real-life system and its replication as a digital twin using system models.

In the context of ZDM, the production process represented by the system model is affected by the following domain aspects:

- $\quad$ The wear and tear of different machine components and parts from a mechanical perspective need to be captured by specific condition-related states in the model.

- Empiric data: rules-of-thumb via observations and experience (which often is undocumented) need to be captured by specific parameters that reflect these aspects.

- Facts and data from production results need to be captured in the outputs generated by the model.

- Underlying understanding of the input qualities, production process, process parameters and output qualities need to be captured in the parameters of the model.

Related questions to the above bullets are: the length of production campaigns or series and how much time passes between the campaigns or series. As the production pace and length of campaigns render wear and tear it is necessary to have a model where both condition- and time-based maintenance are present. Additional external parameters, such as scheduling and re-scheduling of production and maintenance, should be part of a future, more advanced, model. An important aspect that needs to be considered is also the operational context of the production process. Most production processes can be understood as discrete production stages that only interact through the product flow within the production chain, while the individual stages in a production process do have a continuous behavior with a high level of interaction between the product and the dynamics of the production stage. As a result, the production process and the associated system model need to represent these different levels, which requires different modeling strategies and approaches. An effective method to distinguish different levels is to apply time scales and the category discrete/continuous. It is important to note that the system model also needs to reflect the context for which the model is derived: operation, maintenance, monitoring, control, production optimization or process stage optimization.

An often overseen aspect in the modeling of production processes is the control system and its intimate connection with the production processes' physical parts. Depending on the time scale, the effect of the control system needs to be considered in the modeling, meaning the modeling has to be conducted in a closed-loop context when data from an operation is considered. 
A generic representation of the closed-loop system where the production process is interconnected with a control system is depicted in Figure 5. There, it is assumed that $x, y_{K}, y_{e}$ and $d$ are available either as their true values or their estimated counterparts. The output $y$ from $P$ is given as $y=\left[y_{e}, y_{K}\right]^{T}$, where only the outputs in the subset $y_{K}$ are used in the feedback control contexts as CVs. Moreover, $r$ represents the command inputs to the complete system in terms of set points, $u_{e}$ are the exogenous input signals that are not the result of a control action, and $u_{K}$ are the MVs acting on the production process and generated by the control system $K$. By appropriately modeling the control system $K$, both open and closed loop control architectures can be reflected, and subsystems acting on different time scales or residing on different hierarchical levels could be aggregated into one and the same framework.

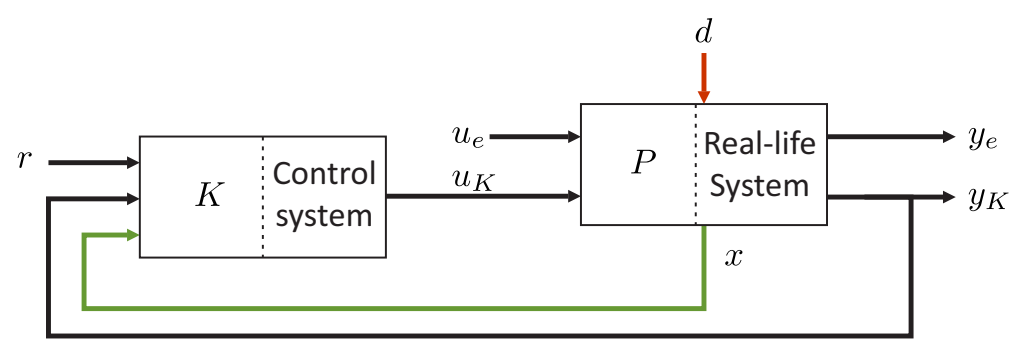

Figure 5. Block diagram representing a generic production process with a connected control system.

\subsection{Realization of a Model Enabling ZDM}

On the basis of the above reasoning, a model enabling ZDM is now derived. Firstly, a generic state space representation for a nonlinear dynamic system is used to model $P$ and $K$ as follows:

$$
\begin{gathered}
P:\left\{\begin{array}{c}
\dot{x}(t)=f_{P}\left(x(t), u_{e}(t), u_{K}(t), d(t), \theta_{f, P}(t)\right) \\
{\left[\begin{array}{l}
y_{e}(t) \\
y_{K}(t)
\end{array}\right]=h_{P}\left(x(t), u_{e}(t), u_{K}(t), d(t), \theta_{h, P}(t)\right)}
\end{array}\right. \\
K:\left\{\begin{array}{l}
\dot{x}_{K}(t)=f_{K}\left(x_{K}(t), r(t), x(t), y_{K}(t), \theta_{f, K}(t)\right) \\
u_{K}(t)=h_{K}\left(x_{K}(t), r(t), x(t), y_{K}(t), \theta_{h, K}(t)\right)
\end{array}\right.
\end{gathered}
$$

For the sake of simplicity and with no loss of generality, the time dependency variable will be dropped. The representations for $P$ and $K$ are complex networks and have dimensions in accordance with the vector signals in the loop, as $r \in R^{n_{r}}, u \in R^{n_{u}}, d \in R^{n_{d}}, y \in R^{n_{y}}$ and $x \in R^{n_{x}}$. There, $n_{r}, n_{u}, n_{d}$, $n_{y}$ and $n_{x}$ are the respective dimensions of the signals. $x_{K}$ denotes the internal states of the control system, and $\theta_{\bullet}$ represents the parameter vectors for the respective function in the system equation. It is also assumed that the production process can be approximated by a non-linear differential equation. The presence of $u_{e}$ and $u_{K}$ in $h_{P}$ constitutes the presence of a direct terms in the real-life system, which is quite uncommon, and will be neglected in the sequel.

It is important to note that the state vector $x$ and output $y$ can reflect both operational and condition-based maintenance aspects of the production process $P$ or $P_{C L}$. If both aspects are considered, then the state vector and output can be segmented into $x=\left[\begin{array}{ll}x_{O} & x_{C}\end{array}\right]^{T}$ and $y=\left[\begin{array}{ll}y_{O} & y_{C}\end{array}\right]^{T}$ by rearranging (1) and (2), respectively. There, $x_{C}$ and $y_{C}$ reflect the production process condition as a result of wear and tear in the process. The feedback signal $y_{K}$ is traditionally only a subset of $y_{O}$. Condition-based maintenance approaches can then be implemented on the basis of the knowledge of $x_{C}$ and $y_{C}$, but require that the model does consider wear and tear aspects.

Clearly, (1) and (2) are connected differential equations through the feedback that is depicted in Figure 5 and can be restated as a differential equation where $r$ and $d$ are the exogenous inputs. In that way the closed-loop system would follow the same structure as given in Figure 3 with an integrated-control system and would constitute a CPS representation. By defining the augmented state 
vector $x_{A}=\left[\begin{array}{ll}x & x_{K}\end{array}\right]^{T}$ and assuming $P$ does not have a direct term, the resulting system equations for the closed loop render

$$
P_{C L}:\left\{\begin{aligned}
\dot{x}_{A} & =\left[\begin{array}{l}
f_{P}\left(x, h_{K}\left(x_{K}, r, x, h_{P}\left(x, d, \theta_{h, P}\right), \theta_{h, K}\right), u_{e}, d, \theta_{f, P}\right) \\
f_{K}\left(x_{K}, r, x, h_{P}\left(x, d, \theta_{h, P}\right), \theta_{f, K}\right)
\end{array}\right] \\
y & =h_{P}\left(x, d, \theta_{h, P}\right)
\end{aligned}\right.
$$

The nonlinear functions $f_{P}$ and $f_{K}$ are of appropriate size depending on the size of the signal vectors and the state vectors, and we introduce the nonlinear function $f=\left[f_{P}(\bullet), f_{K}(\bullet)\right]^{T}$. In (3) it can also be seen that $r$ and $d$ are the new exogenous inputs to the system and that $r$ is not directly reflected in the output equation for $y$.

Moreover, the structure of $f_{P}, h_{P}$ and $h_{K}$ determines to what degree different actions of the control system will have an effect on the condition of production process. When it comes to the performance specifications, those are usually reflected through the design of the control system and the operating conditions given by the set points $r$.

\subsection{Reflecting Interconnected Large-Scale Systems}

The generic model $P_{C L}$ can be used to approximate the behavior of physically entangled CPSs. When several such systems are interconnected in a production chain at a producer site or multiple producers in a supply chain, individual $P_{C L}$ can be interconnected into a larger system description.

To this end, assume there are $m$ individual production processes $P_{C L}$ denoted as $P_{C L}^{(i)}$ with $i \in[1, m]$, and that the system $P_{C L}$ can be approximated by a partial linear system in accordance with [38] or an input affine non-linear system [39]. Further, it will be assumed that the input $u_{e}$ can be split into two components $\left[u_{e, l}, u_{e, g}\right]^{T}$, where $u_{e, l}$ are known local exogenous inputs and $u_{e, g}$ are known global exogenous inputs. The global exogenous inputs can thus be outputs from other processes. An approximation of (3) as a input-affine non-linear system is given as

$$
P_{C L}^{\prime}:\left\{\begin{array}{l}
\dot{x}_{A}=f\left(x_{A}, \theta\right)+g_{l}\left(x_{A}, \theta\right)\left[u_{e, l}, r, d\right]^{T}+g_{g}\left(x_{A}, \theta\right) u_{e, g} \\
y=h\left(x_{A}, \theta\right)+h_{d}\left(x_{A}, \theta\right) d
\end{array}\right.
$$

where $\theta=\left[\theta_{h, P}, \theta_{h, K}, \theta_{f, K}\right]^{T}, g_{l}$ is the local input function, $g_{g}$ is the global input function, and $h_{d}$ is the local disturbance output function. Here, $h_{d}$ is assumed to be zero. Using (4) the combined Large-Scale (LS) system description can be stated as

$$
\begin{aligned}
& {\left[\begin{array}{c}
\dot{x}_{A}^{(1)} \\
\dot{x}_{A}^{(2)} \\
\vdots \\
\dot{x}_{A}^{(m)}
\end{array}\right] }=\left[\begin{array}{cccc}
f^{(1)} & g_{g}^{(1,2)} h^{(2)} & \cdots & g_{g}^{(1, m)} h^{(m)} \\
g_{g}^{(2,1)} h^{(1)} & f^{(2)} & \cdots & g_{g}^{(2, m)} h^{(m)} \\
\vdots & \vdots & \ddots & \vdots \\
g_{g}^{(m, 1)} h^{(1)} & g_{g}^{(m, 2)} h^{(2)} & \cdots & f^{(m)}
\end{array}\right]+\left[\begin{array}{c}
g_{l}^{(1)}\left[u_{e, l}^{(1)}, r^{(1)}, d^{(1)}\right]^{T} \\
g_{l}^{(2)}\left[u_{e, l}^{(2)}, r^{(2)}, d^{(2)}\right]^{T} \\
\vdots \\
g_{l}^{(m)}\left[u_{e, l}^{(m)}, r^{(m)}, d^{(m)}\right]^{T}
\end{array}\right] \\
& {\left[\begin{array}{c}
y^{(1)} \\
y^{(2)} \\
\vdots \\
y^{(m)}
\end{array}\right]=\left[\begin{array}{cccc}
h_{P}^{(1)} & 0 & \cdots & 0 \\
0 & h_{P}^{(2)} & \cdots & 0 \\
\vdots & \vdots & \ddots & \vdots \\
0 & 0 & \cdots & h_{P}^{(m)}
\end{array}\right] }
\end{aligned}
$$

where arguments are omitted for the sake of clarity and the interconnections are represented by the nonlinear functions $g_{g}^{(i, j)}$. In cases where all outputs $y^{(j)}$ are forwarded unaltered from system $P_{C L}^{(j)}$ to $P_{C L}^{(i)}$, the function $g_{g}^{(i, j)}$ does not select specific outputs. Otherwise, the function $g_{g}^{(i, j)}$ might be applied to $h_{P}^{(j)}$, which selects and alters certain measurable outputs.

In (5), material and information flows can be modeled by appropriately defining the interconnection functions $g$. It is also possible to realize sequential and circular dependencies between production 
processes. When the interconnections contain a dynamic behavior themselves, then an intermediate production process needs to be created that contains the states of the interconnection. A typical example for such an interconnection is storage facilities that are used in the interconnection, or when estimators are used to derive information on the connected production processes states.

In Figure 6, an example for a large production system with five unit processes as subsystems is considered. The five subsystems are part of a sequential production chain, where the forward flow and interaction between the unit processes is indicated by the outputs that are forwarded to the individual unit processes. It can be seen that the outputs of $P_{C L}^{(3)}$ and $P_{C L}^{(5)}$ are split into two subsets $A$ and $B$. The subset $A$ is always forwarded to the next process, while subset $B$ is recycled or returned to a previous process in the chain. The resulting model $P_{C L}^{L S}$ can then be stated as follows in (6):

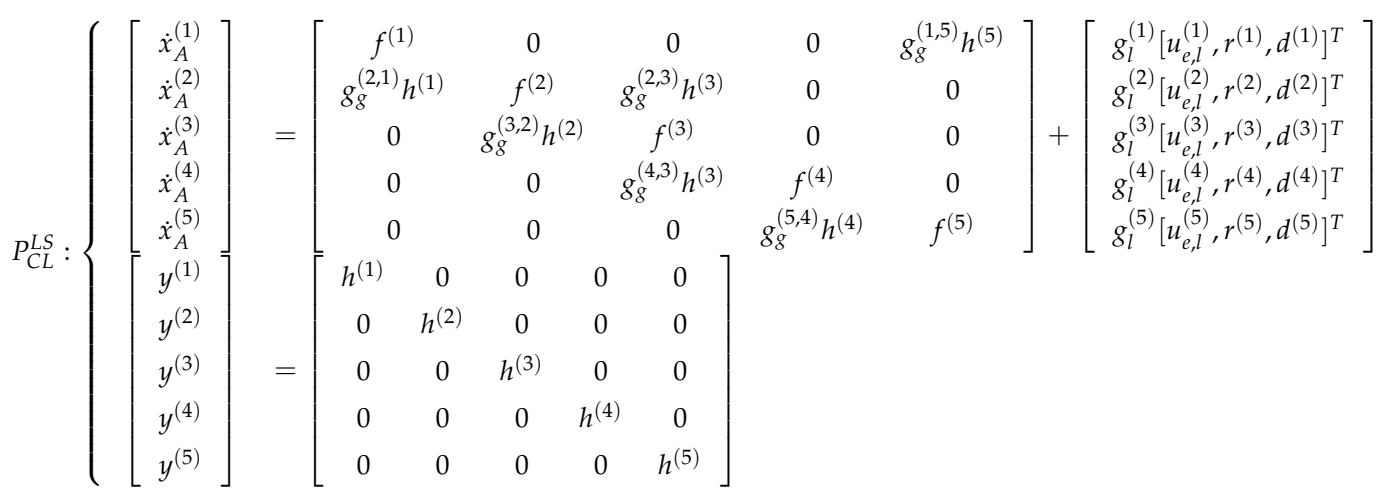

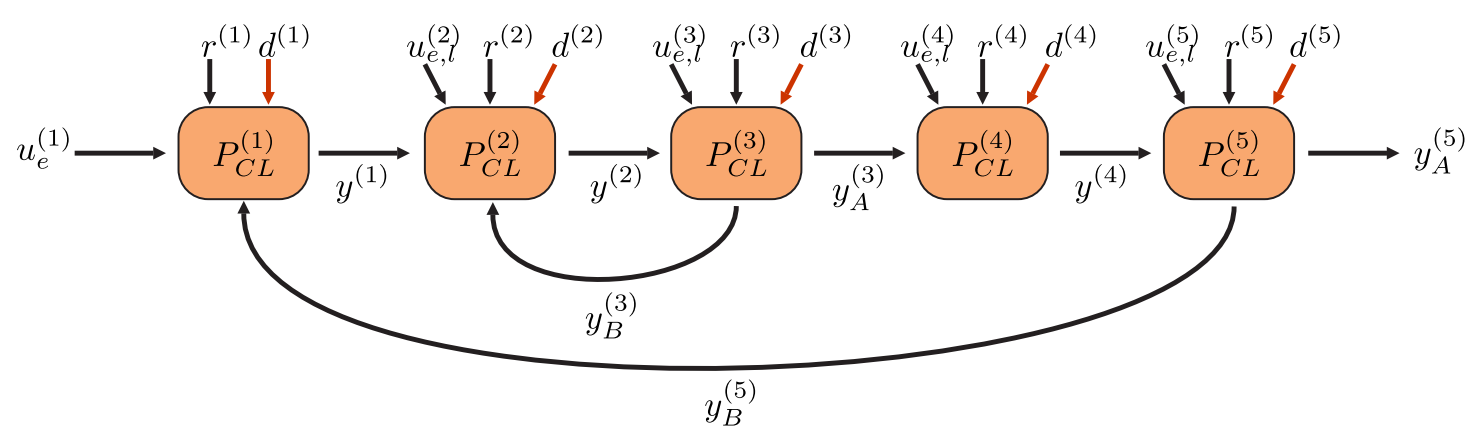

Figure 6. Example for a large system $P_{C L}^{L S}$ with five unit processes in a sequential production chain with two recycle flows between two subsystems.

It is now important to consider whether the suggested modeling approach will be able to capture the model aspects for ZDM. Since the model $P_{C L}$ covers the interplay between condition and operation, the wear and tear aspects are then well considered. Furthermore, all the data that are associated with the empirical understanding of the process and its degradation are usually reflected in either the parameters of the model or the measurements that are acquired. The process performance is usually reflected in some of the measurement variables that are used to assess the production result and establish output qualities. There is also an interplay between the production process specification and requirements and the process performance, which is reflected in the model parameters. Input qualities are also reflected through the effects on the model states through either $u$ or $d$, depending on if the input quality can be controlled.

\subsection{Optimization for ZDM}

An obvious advantage of having $P_{C L}$ available is the possibility to optimize operation while considering the production process condition. If the outputs $y_{O}$ also reflect product quality, then the operation can be optimized towards ZDM. 
A general optimization problem can thus be stated as

$$
\begin{array}{rl}
{\left[r^{*}, y_{O}^{*}, y_{C}^{*}\right]=\arg \min _{r}} & J\left(y_{O}, y_{C}\right) \\
\text { s.t. } & P_{C L} \\
& \underline{C}_{y_{O}}<y_{O}<\bar{C}_{y_{O}} \\
& \underline{C}_{y_{C}}<y_{C}<\bar{C}_{y_{C}}
\end{array}
$$

The cost function $J$ reflects the trade-off between operation and the condition of the production process, where $y_{O}$ represents the quality of the product and $y_{C}$ the condition of the production process. The constraints $C_{\bullet}$, with their respective upper and lower values, enforce quality constraints on the product, but also safety aspects on the operation of the production process through the condition output $y_{0}$.

\section{Analysis}

The initial model for ZDM outlined in Section 4 is intended to be generic and adaptable primarily for manufacturing but also for process industry companies. As stated in the introduction, our view on ZDM is based on our previous research $[3,7,8]$, and combines and integrates seven areas analyzed towards the initial model:

- Monitoring of process parameters-the model in (1) provides an estimate for measured process parameters and in turn can be used to monitor the deviation between actual and desired values.

- Collaborative manufacturing (which includes sharing of manufacturing data without media breaks along the supply chain)—-the system equations and system descriptions given in (3), (5) and (6) can be a combination of several interconnected sub-systems that reflect the individual unit operation and the interconnections. In that way, it is even possible to reflect supply chains in an overarching system model that contains the bi- or multilateral information flow between individual unit operations or manufacturing units.

- Continuous quality control (which includes measurement of input to the process and measurement of output from the process with automatic sorting) - quality control depends on the sampling and assessment of the produced components, which are not directly related to the system model. However, quality variations can be related to changes in the process and potential faults needing to be addressed. It is therefore of importance to not only represent the process in terms of its operational characteristics and degradation, but also how the process nominally leads to product qualities on the basis of input material properties.

- On-line predictive maintenance-the system equations in (3) do contain states reflecting both the condition and operation of a process and are used to optimize process operation as stated in (7). Predictive maintenance relies on the proper prediction of the degradation of a process in relation to the operation of a process. It is thereby possible to provide an estimate of the remaining useful life of components in a process and to act proactively.

- Data storage, analytics and visualization - the modeling approach described does not directly provide specific methodologies for storage or visualization, but is an integral part of the analytics by providing the needed predictions and estimates for process variables and process parameters. Analytics is usually an important component of any decision support solution for operation and maintenance.

- Re-configuration and re-organization of production-a needed insight for these operational decisions is the condition of the process. Early detection of incipient faults is an essential understanding for production decision making. For this end, different production scenarios and their impact on both operation and degradation are integral aspects when tailoring the methodology to a specific case.

- Re-scheduling of production-a basic requirement to have re-scheduling capability is the availability of additional production resources or timely flexibility. In conjunction with a predictive 
maintenance scheme, flexibility enables alignment of maintenance needs, operational needs and order flows. Ideally, this enables increased production system utilization and overall equipment efficiency.

The reason for combining and integrating the seven areas is that when they are combined and integrated, a better result can be achieved [8]. Based on the analysis above, we posit that this can be done using our initial model for ZDM.

In a practical manufacturing perspective at Gestamp HardTech AB (cf. [8]), the first five of the seven areas above are currently being addressed and the last two will soon be taken into consideration. A complete system model, i.e., digital twin, needs to be further developed. The development of systems for inline and high-accuracy geometry measurements of all output quality is progressing well. The continuous and accurate measurements of the output are key to the whole ZDM approach and model. Currently, the last two of the seven areas are manually decided based on multiple sources of information and experience.

Further, in a theoretical perspective, Figure 7 outlines how much investments into new machinery (CAPEX) and increased operational support (OPEX), such as maintenance, repair and overhaul, relate to the wanted level of output quality (parts or products). The CAPEX and OPEX can be varied as long as the output quality is within specifications and meets the customers' requirements-and should be optimized for that in order to not have an unnecessarily high total cost level. Thus, it is necessary to know where the optimum for the cost function is in order to remain close to it and maximize the output quality toward the related costs, while also taking into account the spread of both the measurement and the underlying quality. The small box in Figure 7, denoted "Target quality vs. cost", indicates the need for a margin above the customer requirement due to measurement errors and uncertainties. Clearly, a better model and improved measurements entail a reduction of the margin and the reduction of the box. As a consequence, a reduced margin is also associated with decreased total cost.

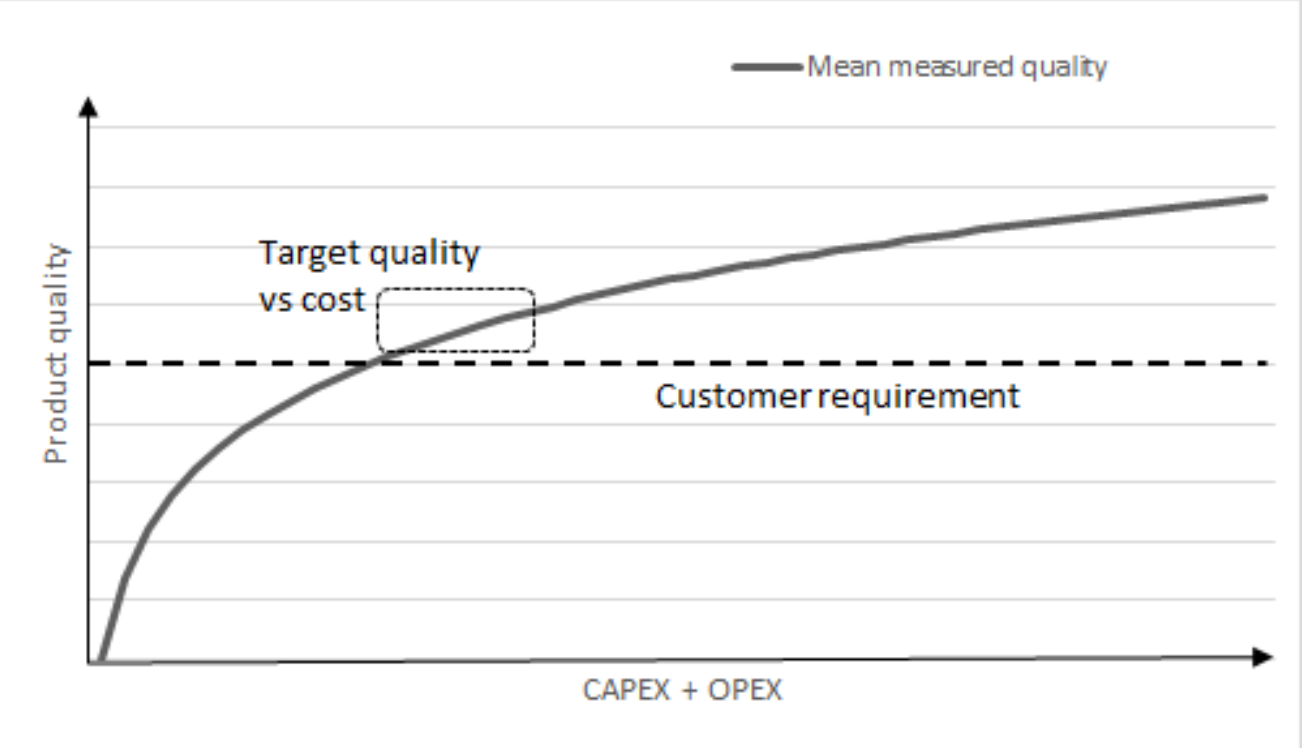

Figure 7. Schematic diagram of how the quality, theoretically, can be seen to relate to investments into new machinery and increased operational support (CAPEX and OPEX).

Concerning the design criteria, i.e., simplicity, low cost, robustness, high-quality output and future-proofing, the initial ZDM model outlined is aligned with these. However, it needs to be tested in order to determine whether it is simple, does not cost a lot to implement, is robust or needs further improvement, and whether it helps to ensure high-quality output and helps to future-proof the companies that will use it. 


\section{Discussion and Conclusions}

The paper contributes to the literature with an initial model for ZDM that can be adapted primarily for manufacturing but also for process industry companies. The initial model is a further development of the ZDM approach previously outlined in [7,8], and a step towards implementation in real cases. Further, the initial ZDM model continues to developing ZDM as a concept via a systematic approach with a solid mathematical foundation.

Further, the paper contributes to practice by bringing up a number of areas and topics that are preconditions for moving towards the ZDM approach and implementation of a ZDM model. The preconditions are, for instance: (i) monitoring of adequate process parameters; (ii) an ability to use data about the input and provide data to the next or previous step(s) in the value chain; (iii) measure relevant parameters on the input and output and potentially also in the process where relevant, so as to be able to sort out what should not be conveyed to the next step; (iv) monitoring of relevant parameters of production equipment status and function and predictive maintenance for the equipment; and (v) data storage with analytics and visualization of results. All five are needed for the initial ZDM model to render full value.

In addition, the managerial contribution of the paper is that the initial ZDM model outlined can be used for: (vi) re-configuration and re-organization of production and (vii) re-scheduling of production.

The system model and connected control systems (see Figures 3 and 4) support both the practical and managerial aspects (i-vii).

The understanding of a system's behavior (see Figures 1 and 2) is crucial in order to model it. Further, it is of utmost importance to take care of not only what can be explained by data but also the domain knowledge, which may be formal (written/documented) or tacit (embodied in production staff, development engineers, maintenance staff, etc.). Without this, the system model will be less useful and less complete.

The initial ZDM model outlined in this paper is a next step and partly a response to what [2,9-11] are looking for. The initial ZDM model further develops the ZDM concept and uses state-of-the-art methods concerning, e.g., fault tolerances, predictive maintenance and continuous quality measurement/monitoring, in order to get to the next level of applicability. The previous barriers for ZDM to be successful (see the introduction section) are starting to be overcome by the recent development of various technologies and methods needed. However, additional development of the initial ZDM model, measurement methods, sensors, cameras etc. is required in order to make ZDM generally available for all manufacturing and process industry companies. The initial ZDM model does not fulfill all posed requirements, but is a step towards the possibility to combine and integrate a number of necessary areas in various industrial settings. Further, the results are deemed as highly generalizable within the manufacturing and process industries as well as critical infrastructures with production and distribution environments. In addition, the possibility to inform the subsequent or previous steps in a production process or value chain enables the integration and interoperability of different systems. A question that needs to be asked is whether these companies will start to address and use ZDM as well as what that requires? Firstly, they need to be made aware and also see the potential, and subsequently they will need to get the knowledge, competence, technology and management support necessary to make these changes in both production processes and attitudes. Not all companies will be able to do so, whereas some will make a step-by-step plan and implement ZDM steadily. It will get easier as ZDM becomes a mainstream approach and have standard toolboxes available.

Concerning future research, we intend to improve, refine and further develop the initial model for ZDM in a number of industrial cases. Planned activities include testing of parts of the ZDM approach and initial model in each of the industrial cases, and aggregating the results into a refined and improved model for ZDM and updating the overall ZDM approach with the new findings.

To conclude, the paper outlines an initial model for ZDM that is highly generalizable and is in line with the desired design criteria. Thus, the ZDM approach outlined in $[7,8]$ can be used together 
with the initial ZDM model to make the production processes of manufacturing and process industry companies more intelligent and improve the companies' sustainability, competitiveness, efficiency and profitability in small steps-which, combined, will render a good effect.

Author Contributions: Conceptualization: J.L., P.K., W.B. and E.L.; Data curation: J.L., P.K. and E.L.; Formal analysis: J.L., P.K. and W.B.; Funding acquisition: P.K.; Investigation: J.L. and P.K.; Methodology: J.L. and W.B.; Project administration: P.K.; Resources: P.K.; Software: W.B.; Supervision: W.B.; Validation: W.B.; Visualization: P.K. and W.B.; Writing—original draft: J.L., P.K. and W.B.; Writing—review \& editing: J.L., P.K., W.B. and E.L. All authors have read and agreed to the published version of the manuscript.

Funding: This research received no external funding.

Conflicts of Interest: The authors declare no conflict of interest.

\section{References}

1. Deming, W.E.; Edwards, D.W. Quality, Productivity, and Competitive Position; Massachusetts Institute of Technology, Center for Advanced Engineering Study: Cambridge, MA, USA, 1982; Volume 183.

2. Psarommatis, F.; May, G.; Dreyfus, P.A.; Kiritsis, D. Zero defect manufacturing: State-of-the-art review, shortcomings and future directions in research. Int. J. Prod. Res. 2020, 58, 1-17. [CrossRef]

3. Lindström, J.; Kyösti, P.; Lejon, E.; Birk, W.; Andersson, A.; Borg, M.; Juntti, M.; Suup, A.M.; Maria Germain, A.H.; Gunnarsson, B. Zero Defect Manufacturing in an Industry4.0 context: A case study of requirements for change and desired effects. Procedia CIRP 2020, in press.

4. Blanke, M.; Kinnaert, M.; Lunze, J.; Staroswiecki, M.; Schröder, J. Diagnosis and Fault-Tolerant Control; Springer: Berlin, Germany, 2006; Volume 2.

5. Leitão, J.; de Brito, S.; Cubico, S. Eco-Innovation Influencers: Unveiling the Role of Lean Management Principles Adoption. Sustainability 2019, 11, 2225. [CrossRef]

6. Horbach, J.; Rammer, C.; Rennings, K. Determinants of eco-innovations by type of environmental impact-The role of regulatory push/pull, technology push and market pull. Ecol. Econ. 2012, 78, 112-122. [CrossRef]

7. Lindström, J.; Larsson, H.; Jonsson, M.; Lejon, E. Towards intelligent and sustainable production: Combining and integrating online predictive maintenance and continuous quality control. Procedia CIRP 2017, 63, 443-448. [CrossRef]

8. Lindström, J.; Lejon, E.; Kyösti, P.; Mecella, M.; Heutelbeck, D.; Hemmje, M.; Sjödahl, M.; Birk, W.; Gunnarsson, B. Towards intelligent and sustainable production systems with a zero-defect manufacturing approach in an Industry4.0 context. Procedia CIRP 2019, 81, 880-885. [CrossRef]

9. Konrad, B.; Lieber, D.; Deuse, J. Striving for zero defect production: Intelligent manufacturing control through data mining in continuous rolling mill processes. In Robust Manufacturing Control; Springer: Berlin, Germany, 2013; pp. 215-229.

10. Lee, J.; Bagheri, B.; Kao, H.A. A cyber-physical systems architecture for industry 4.0-based manufacturing systems. Manuf. Lett. 2015, 3, 18-23. [CrossRef]

11. Lin, Y.C.; Hung, M.H.; Huang, H.C.; Chen, C.C.; Yang, H.C.; Hsieh, Y.S.; Cheng, F.T. Development of advanced manufacturing cloud of things (AMCOT) - A smart manufacturing platform. IEEE Robot. Autom. Lett. 2017, 2, 1809-1816. [CrossRef]

12. Zairi, M. Beyond TQM implementation: The new paradigm of TQM sustainability. Total Qual. Manag. 2002, 13, 1161-1172. [CrossRef]

13. Harari, O. Ten reasons why TQM doesn't work. Manag. Rev. 1993, 82, 33.

14. Pyzdek, T. The Six Sigma; Mcgraw-Hill: New York, NY, USA, 2003.

15. Kyösti, P.; Reed, S. Prediction of service support costs for functional products. Simul. Model. Pract. Theory 2015, 59, 52-70. [CrossRef]

16. Lindström, J.; Kyösti, P.; Delsing, J. European Roadmap for Industrial Process Automation. 2018. Available online: https://www.diva-portal.org/smash/record.jsf?pid=diva2\%3A1245908\&dswid=mainwindow (accessed on 1 June 2020).

17. Dong, W.; Liu, S.; Fang, Z.; Yang, X.; Hu, Q.; Tao, L. Control and optimization of quality cost based on discrete grey forecasting model. In Proceedings of the 2017 International Conference on Grey Systems and Intelligent Services (GSIS), Stockholm, Sweden, 8-11 August 2017; p. 149. 
18. Thangaiah, I.S.; Sharma, V.; Sundharam, V.N. Analysing of customer feedback on critical quality parameters to improve productivity in manufacturing-a case study. Int. J. Product. Qual. Manag. 2018, 23, 349-368. [CrossRef]

19. Mourtzis, D.; Vlachou, E.; Milas, N. Industrial Big Data as a result of IoT adoption in manufacturing. Procedia Cirp 2016, 55, 290-295. [CrossRef]

20. Chien, C.F.; Hsu, S.C.; Chen, Y.J. A system for online detection and classification of wafer bin map defect patterns for manufacturing intelligence. Int. J. Prod. Res. 2013, 51, 2324-2338. [CrossRef]

21. Kuo, C.F.; Hsu, C.T.M.; Fang, C.H.; Chao, S.M.; Lin, Y.D. Automatic defect inspection system of colour filters using Taguchi-based neural network. Int. J. Prod. Res. 2013, 51, 1464-1476. [CrossRef]

22. Choi, G.; Kim, S.H.; Ha, C.; Bae, S.J. Multi-step ART1 algorithm for recognition of defect patterns on semiconductor wafers. Int. J. Prod. Res. 2012, 50, 3274-3287. [CrossRef]

23. Ahuett-Garza, H.; Kurfess, T. A brief discussion on the trends of habilitating technologies for Industry 4.0 and Smart manufacturing. Manuf. Lett. 2018, 15, 60-63. [CrossRef]

24. Bergström, P.; Fergusson, M.; Folkesson, P.; Runnemalm, A.; Ottosson, M.; Andersson, A.; Sjödahl, M. Automatic in-line inspection of shape based on photogrammetry. In Proceedings of the 7th Swedish Production Symposium, Lund, Sweden, 25-27 October 2016.

25. Bergström, P.; Fergusson, M.; Sjödahl, M. Virtual projective shape matching in targetless CAD-based close-range photogrammetry for efficient estimation of specific deviations. Opt. Eng. 2018, 57, 053110. [CrossRef]

26. Lejon, E.; Kyösti, P.; Lindström, J. Machine learning for detection of anomalies in press-hardening: Selection of efficient methods. Procedia CIRP 2018, 72, 1079-1083. [CrossRef]

27. Johansson, A.; Birk, W.; Medvedev, A. Model-based gas leakage detection and isolation in a pressurized system via Laguerre spectrum analysis. In Proceedings of the 1998 IEEE International Conference on Control Applications (Cat. No. 98CH36104), Trieste, Italy, 4 September 1998; Volume 1, pp. 212-216.

28. Birk, W.; Johansson, A.; Medvedev, A. Model based control for a fine coal injection plant. IEEE Control. Syst. Mag. 1999, 19, 33-43.

29. Verheyleweghen, A.; Jäschke, J. Framework for combined diagnostics, prognostics and optimal operation of a subsea gas compression system. IFAC-PapersOnLine 2017, 50, 15916-15921. [CrossRef]

30. Reason, P.; Bradbury, H. Handbook of Action Research: Participative Inquiry and Practice; Sage: Thousand Oaks, CA, USA, 2001.

31. Gummesson, E. Qualitative Methods in Management Research; Sage: Thousand Oaks, CA, USA, 2000.

32. Coghlan, D.; Coughlan, P.; Brennan, L. Organizing for research and action: Implementing action researcher networks. Syst. Pract. Action Res. 2004, 17, 37-49. [CrossRef]

33. Remenyi, D. Field Methods for Academic Research: Interviews; Academic Conferences Limited: Sonning Common, UK, 2012.

34. Patton, M.Q. Qualitative Evaluation and Research Methods; Sage: Thousand Oaks, CA, USA, 1990.

35. Kvale, S.; Brinkmann, S. Interviews: Learning the Craft of Qualitative Research Interviewing; Sage: Thousand Oaks, CA, USA, 2009.

36. Fontana, A.; Frey, J.H.; Denzin, N.K.; Lincoln, Y.S. Handbook of Qualitative Research; Norman, K., Yvonna, S., Eds.; Sage: London, UK, 1994.

37. Milles, M.B.; Huberman, A.M. An Expanded Sourcebook: Qualitative Data Analysis; Sage: Thousand Oaks, CA, USA, 1994; Volume 12, p. 2016.

38. Mazenc, F. Interconnected nonlinear systems, local and global stabilization. Syst. Control Lett. 1998, 35, 317-323. [CrossRef]

39. Sontag, E.D. Mathematical Control Theory: Deterministic Finite Dimensional Systems; Springer Science \& Business Media: Berlin, Germany, 2013; Volume 6.

(C) 2020 by the authors. Licensee MDPI, Basel, Switzerland. This article is an open access article distributed under the terms and conditions of the Creative Commons Attribution (CC BY) license (http:/ / creativecommons.org/licenses/by/4.0/). 